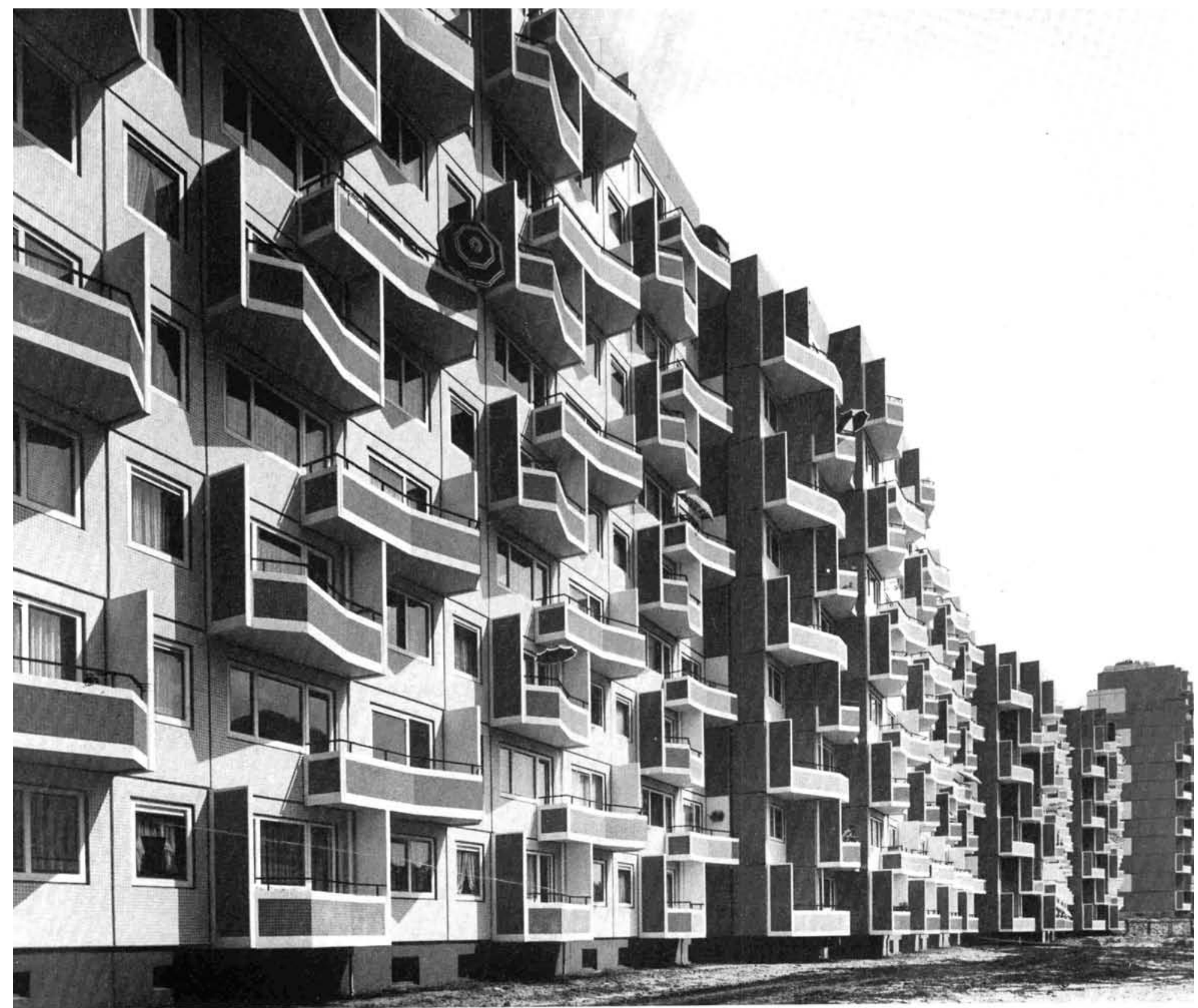

simopesis

El conjunto erigido -21 casas con un total de 1.061 viviendas- consta de dos unidades: una de 1.061 viviendas- consta de druidas con el sistema de prefabricací́n «Barets»; y la otra con 345 vide prefabricanion bos sistemas a base de paneles macizos - realiza. dos en hermigón armado- en muros interiores forjados; $y$ de paneles tipo "Sandwich" en cerra. mientos exteriores.

Digna de mención es la construcción de los balco. nes, concebidos y realizados como elementos sus. tentados en el espacio, y colgados de las fachadas. por medio de ménsulas y elementos metálicos. Igualmente cabe destacar la variedad de plantas y alzados y el extraordinario dinamismo y movi. miento del conjunto.

\section{rascacielos, en Hamburgo}

F TRAUTWEIN, Arquitecto: Prof. Dipl. Ingeniero

y R. BEHN, Arquitecto BDA

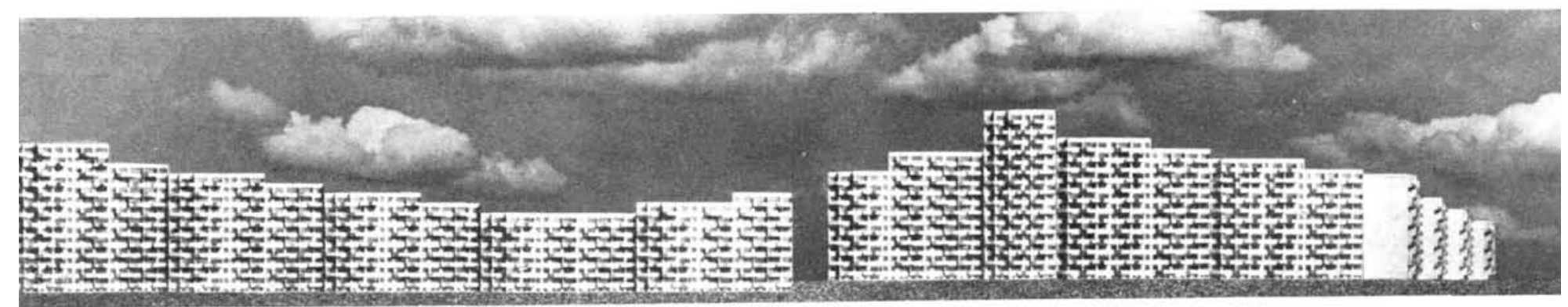




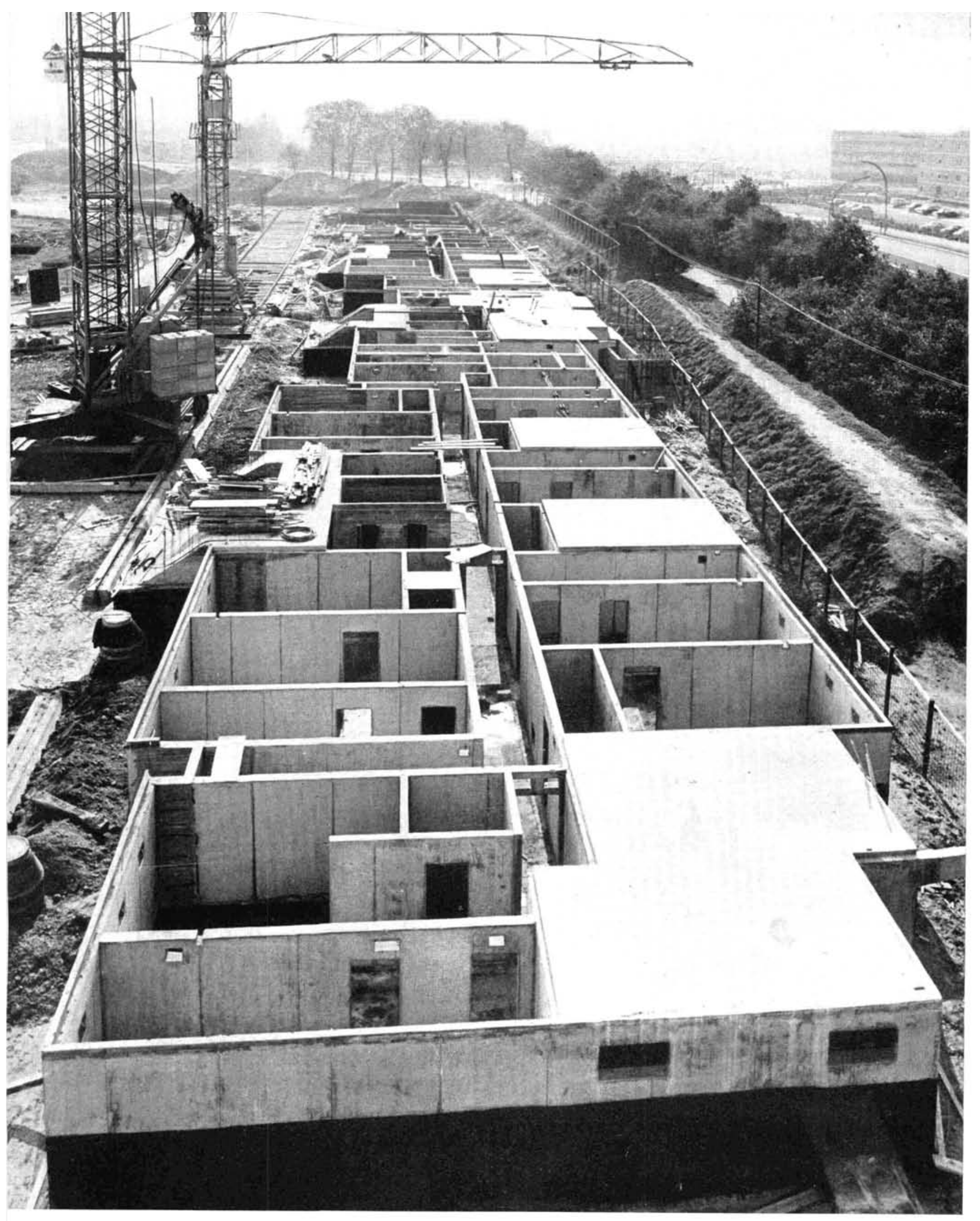

Al recibir el encargo del proyecto de construcción de estas viviendas, a desarrollar sobre un terreno sito en el centro de un sector de nuevas edificaciones - el Osdorfer Born-, los arquitectos estudiaron cuidadosamente la ordenación de bloques y la urbanización general, diseñando en medio de los bloques aislados circundantes - de 3 a 4 plantas-, edificios con una variada distribución de alturas (6 a 21 plantas) y de profundidades. 


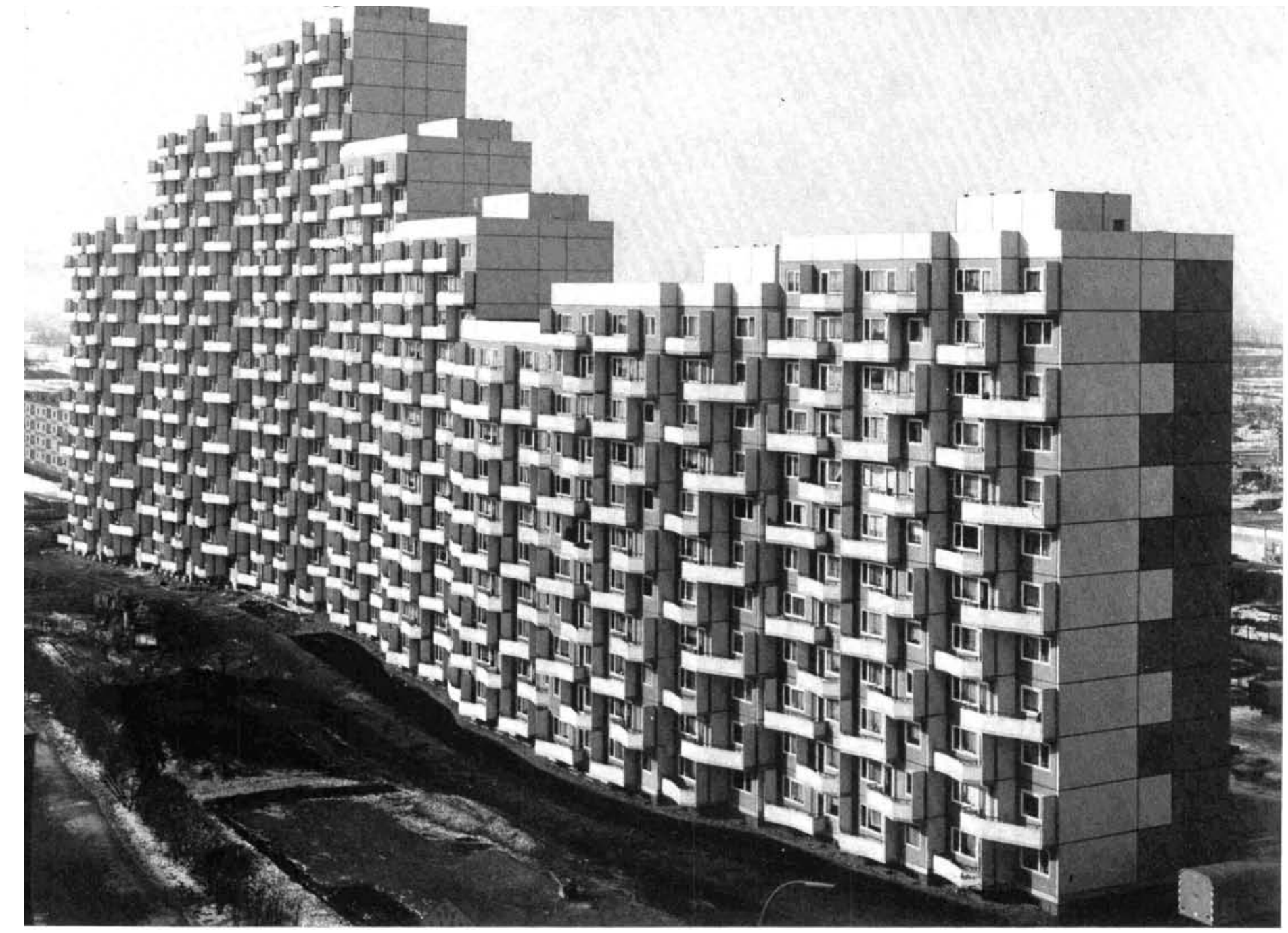

En resumen, el conjunto erigido consta de dos unidades: una de ellas con 716 viviendas, construida por el sistema "Barets»; y la otra, con 345 viviendas, por el sistema «Camus»; la diferencia entre ambos sistemas es insignificante y está localizada en las juntas de unión de los diferentes elementos. Mientras que en las unidades construidas por el sistema "Camus", las piezas fueron transportadas a la obra desde una fábrica situada fuera de ella; en las del sistema "Barets», se utilizaron paneles fabricados en obra en una fábrica portátil — dotada de un gran equipo de maquinaria fácilmente desmontable y trasladable, y de instalaciones automáticas de mezclado y vertido de hormigón.

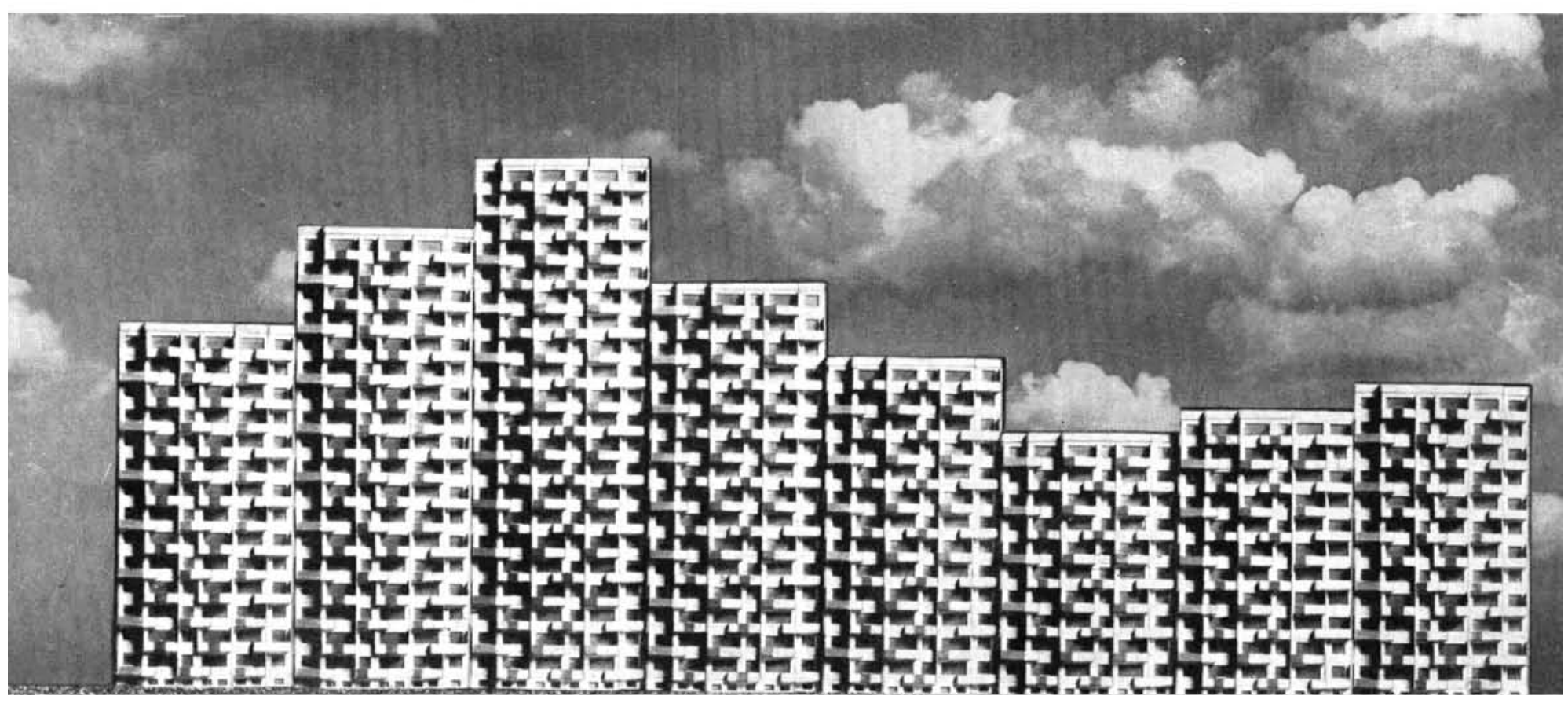




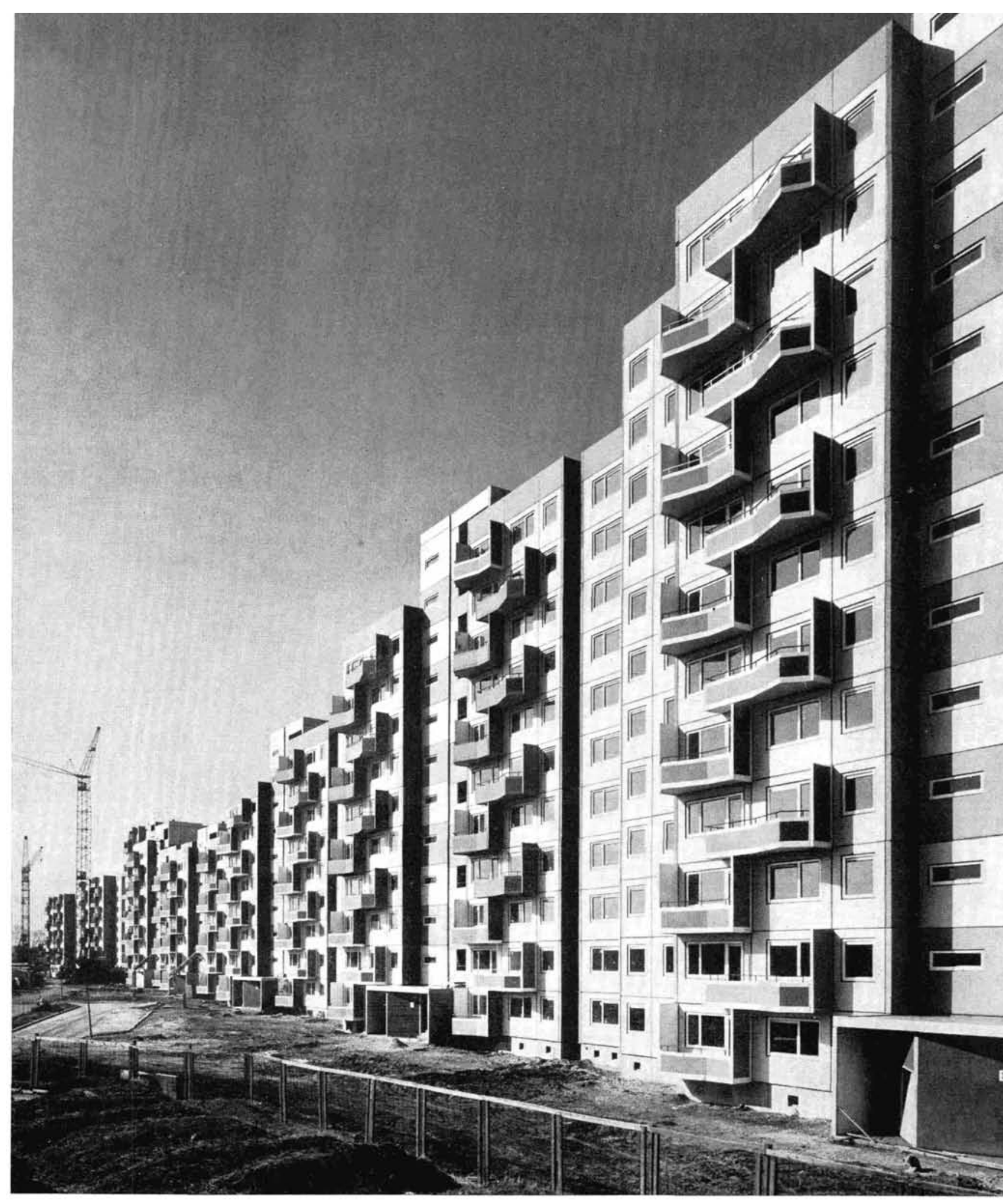

del principio de que los gastos de entretenimiento y limpieza fueran mínimos - realidad a tener en cuenta mucho más en los «rascacielos» que en construcciones comparativamente más bajas-; así, las fa. chadas fueron revestidas con mosaico cerámico fino que, además de ofrecer un buen aspecto estético, satisfacía los mencionados requisitos. En cuanto a la elección de colores, se escogió el blanco para todas las superficies dominantes, en contraste con las superficies de mosaico en azul-gris y blanco-gris y con el mosaico vidriado pequeño, con gran variedad de colores, que aparece en las plantas bajas. 

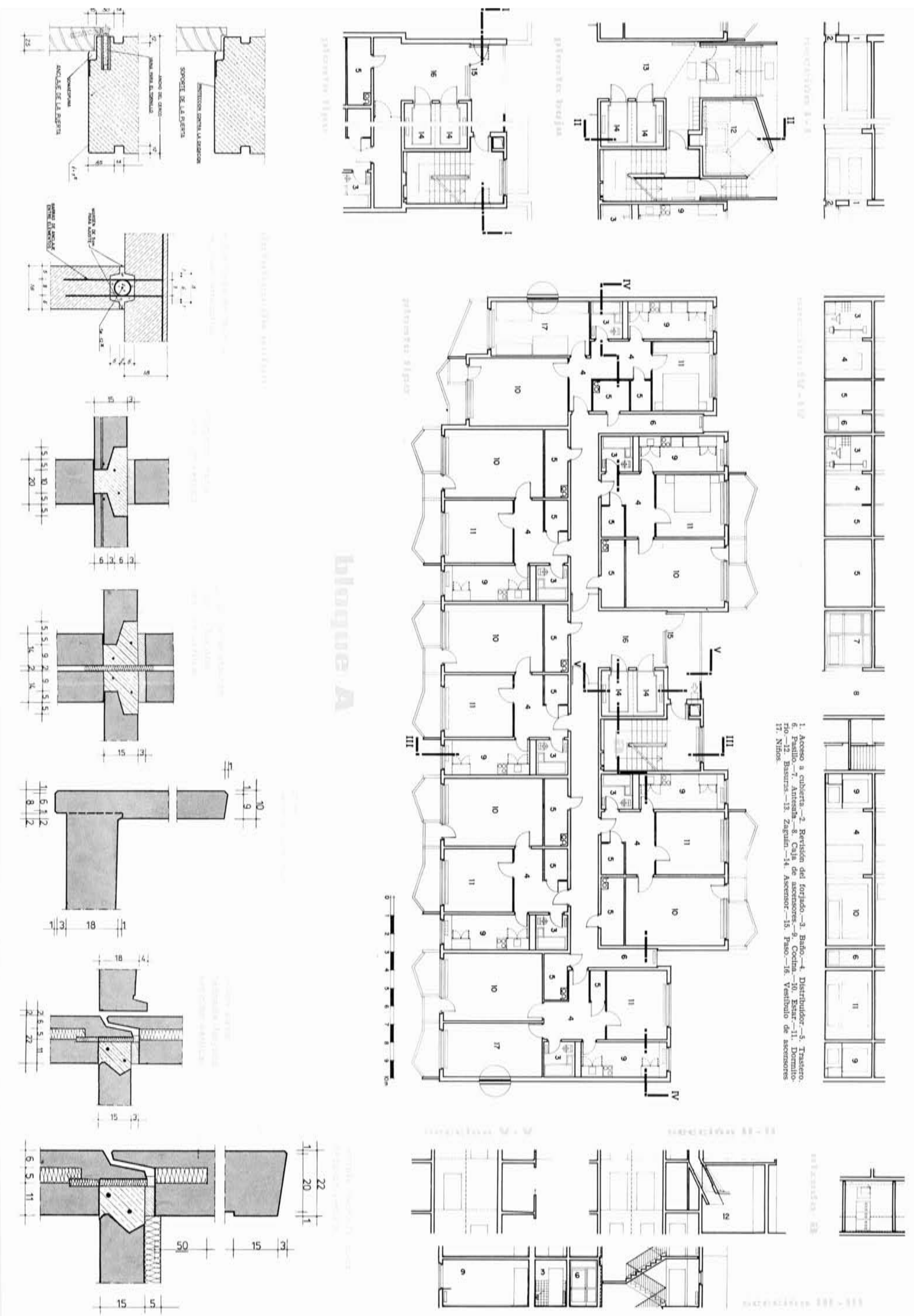


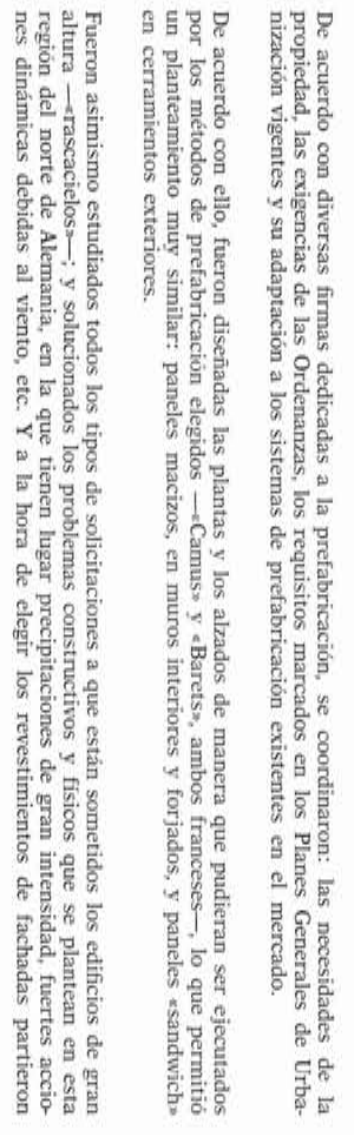
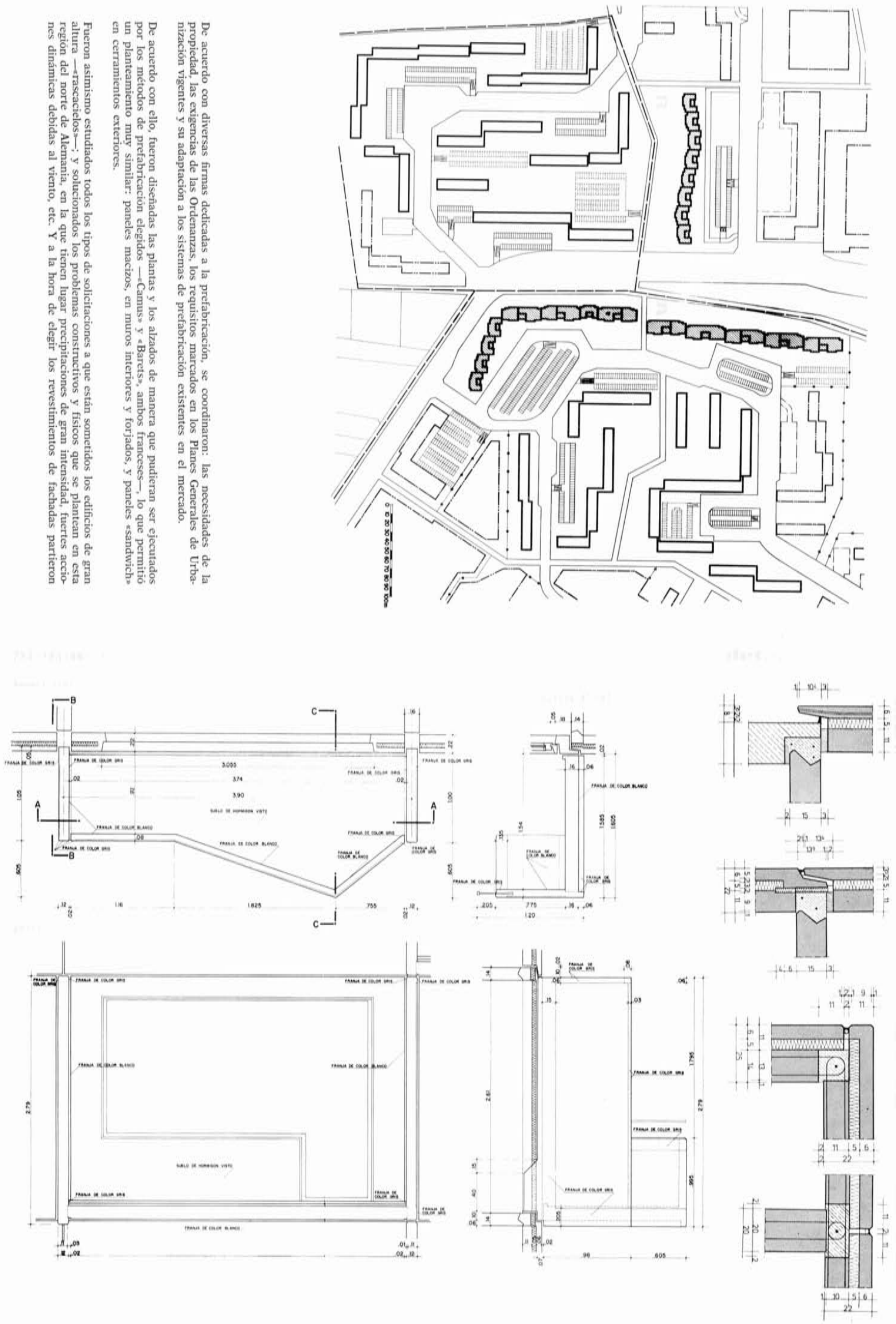

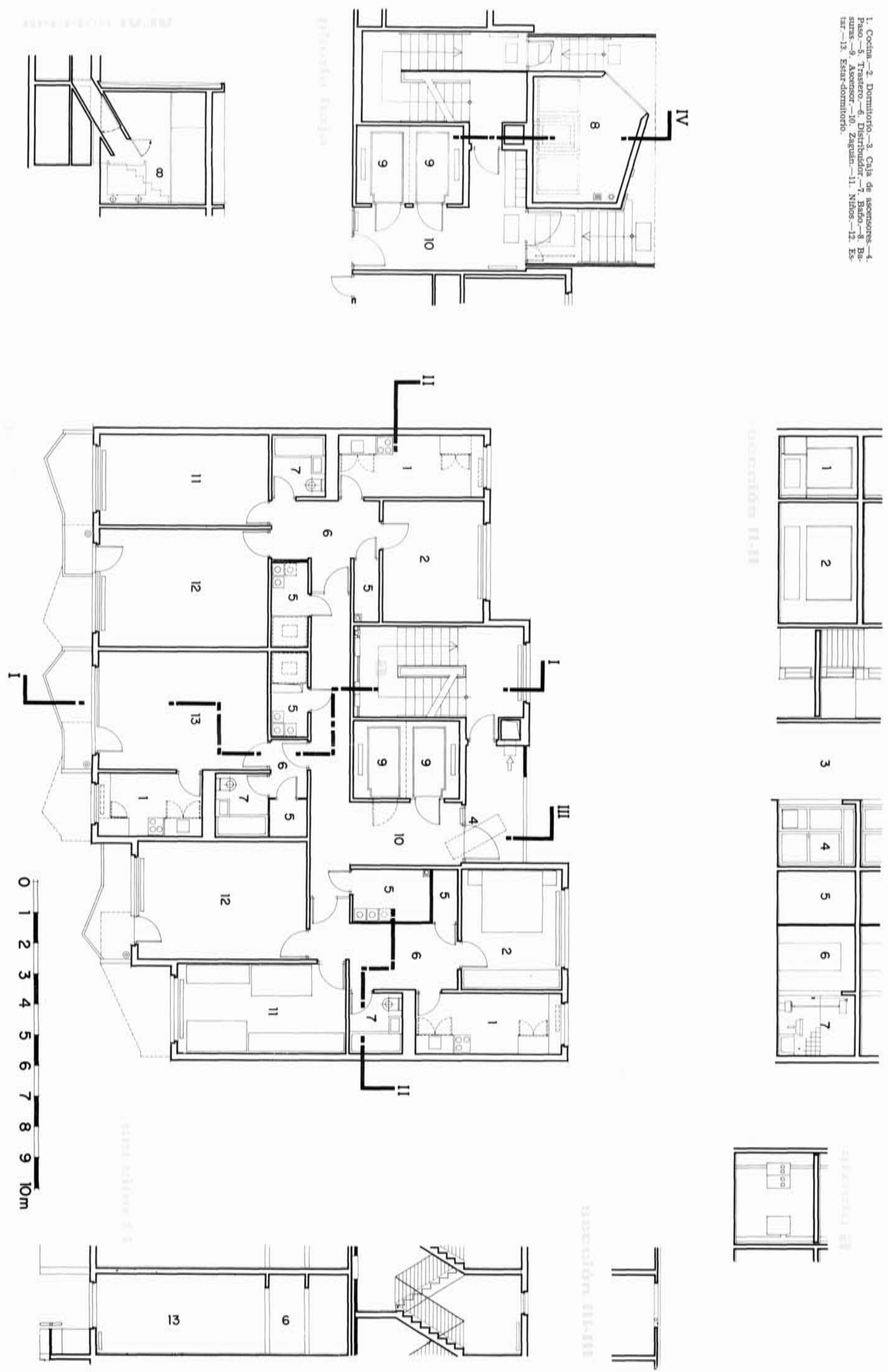

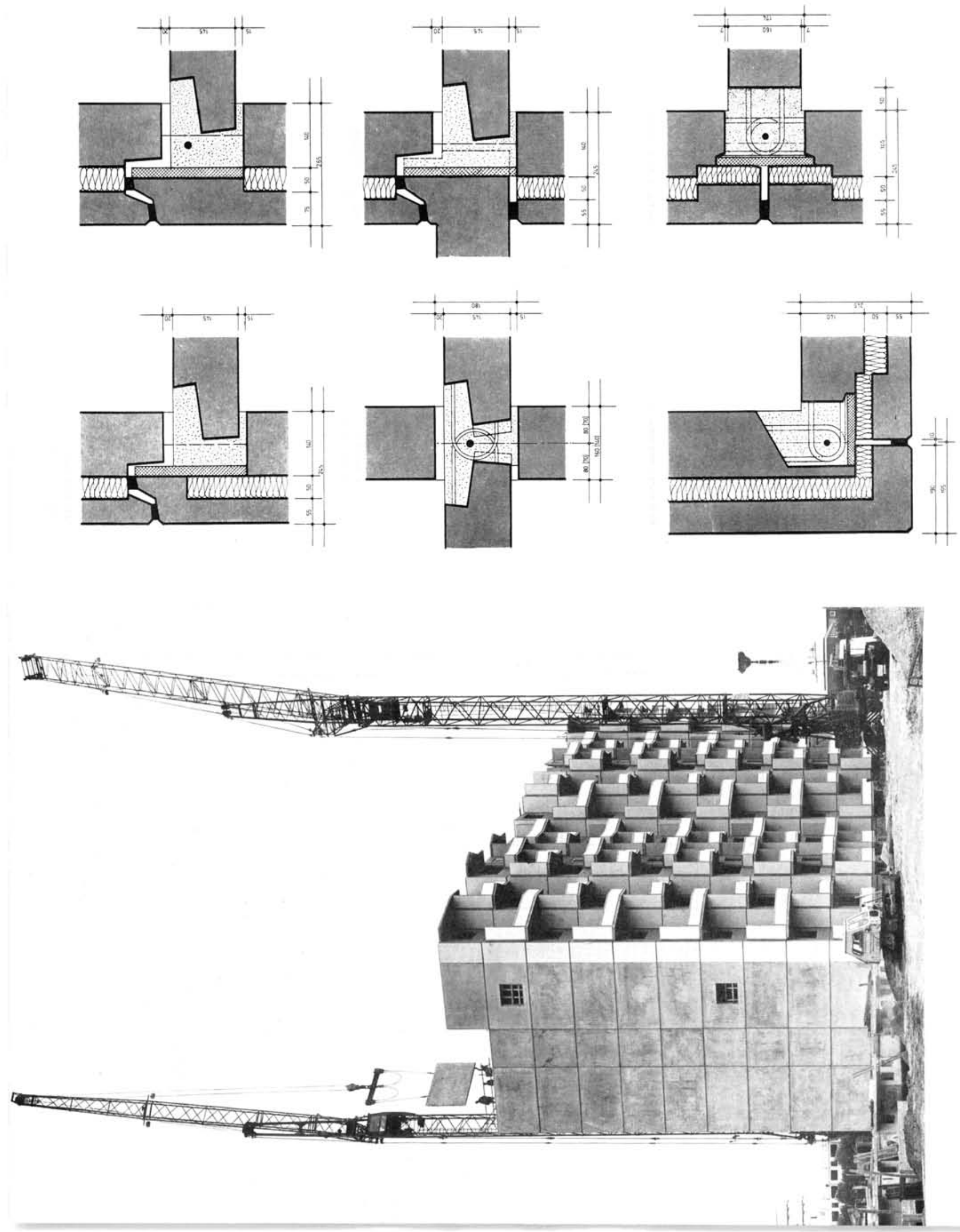

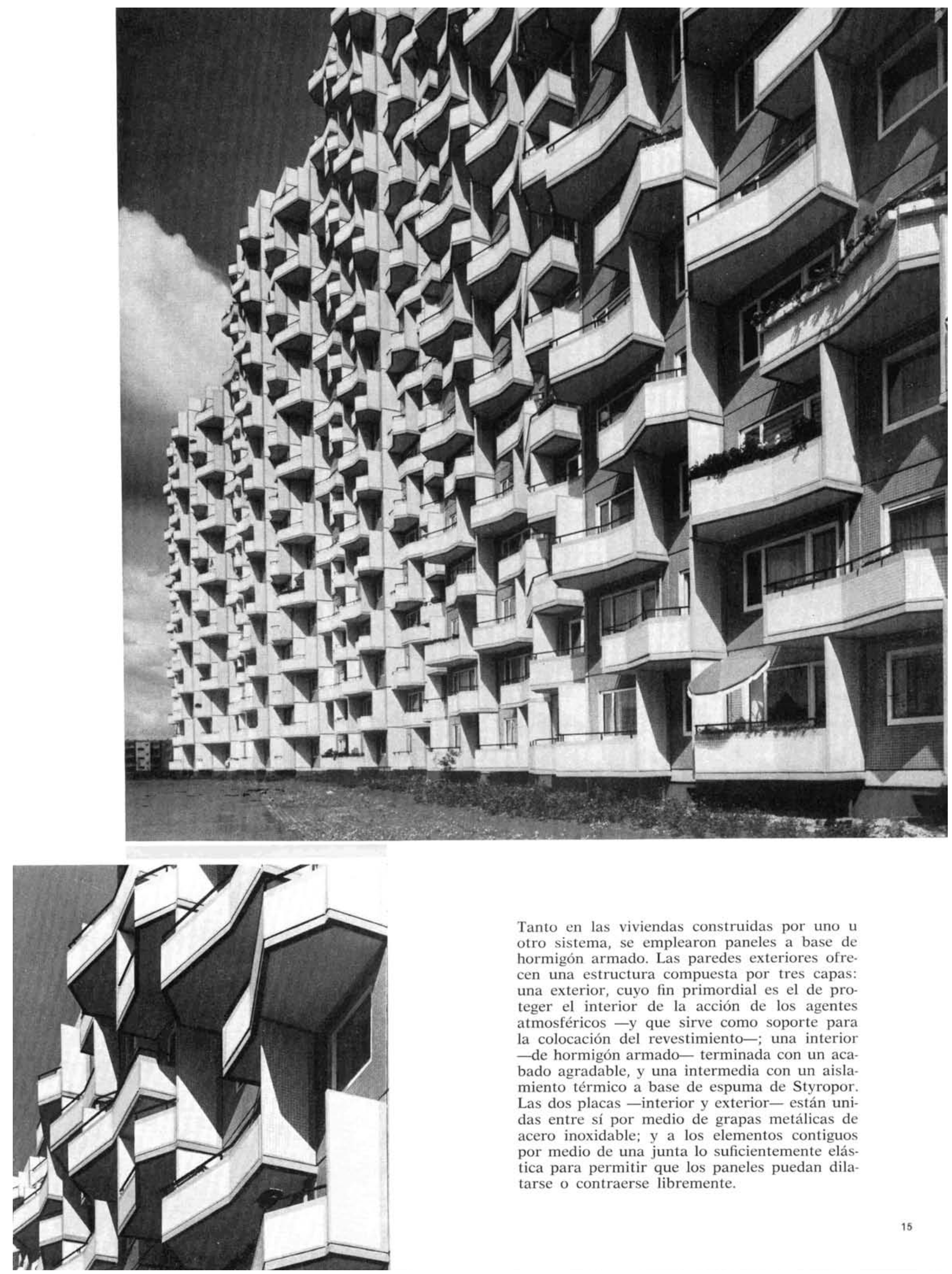

Tanto en las viviendas construidas por uno $\mathrm{u}$ otro sistema, se emplearon paneles a base de hormigón armado. Las paredes exteriores ofrecen una estructura compuesta por tres capas: una exterior, cuyo fin primordial es el de proteger el interior de la acción de los agentes atmosféricos $-\mathrm{y}$ que sirve como soporte para la colocación del revestimiento-; una interior - de hormigón armado - terminada con un acabado agradable, y una intermedia con un aislamiento térmico a base de espuma de Styropor. Las dos placas -interior y exterior- están unidas entre sí por medio de grapas metálicas de acero inoxidable; y a los elementos contiguos por medio de una junta lo suficientemente elástica para permitir que los paneles puedan dilatarse o contraerse libremente. 


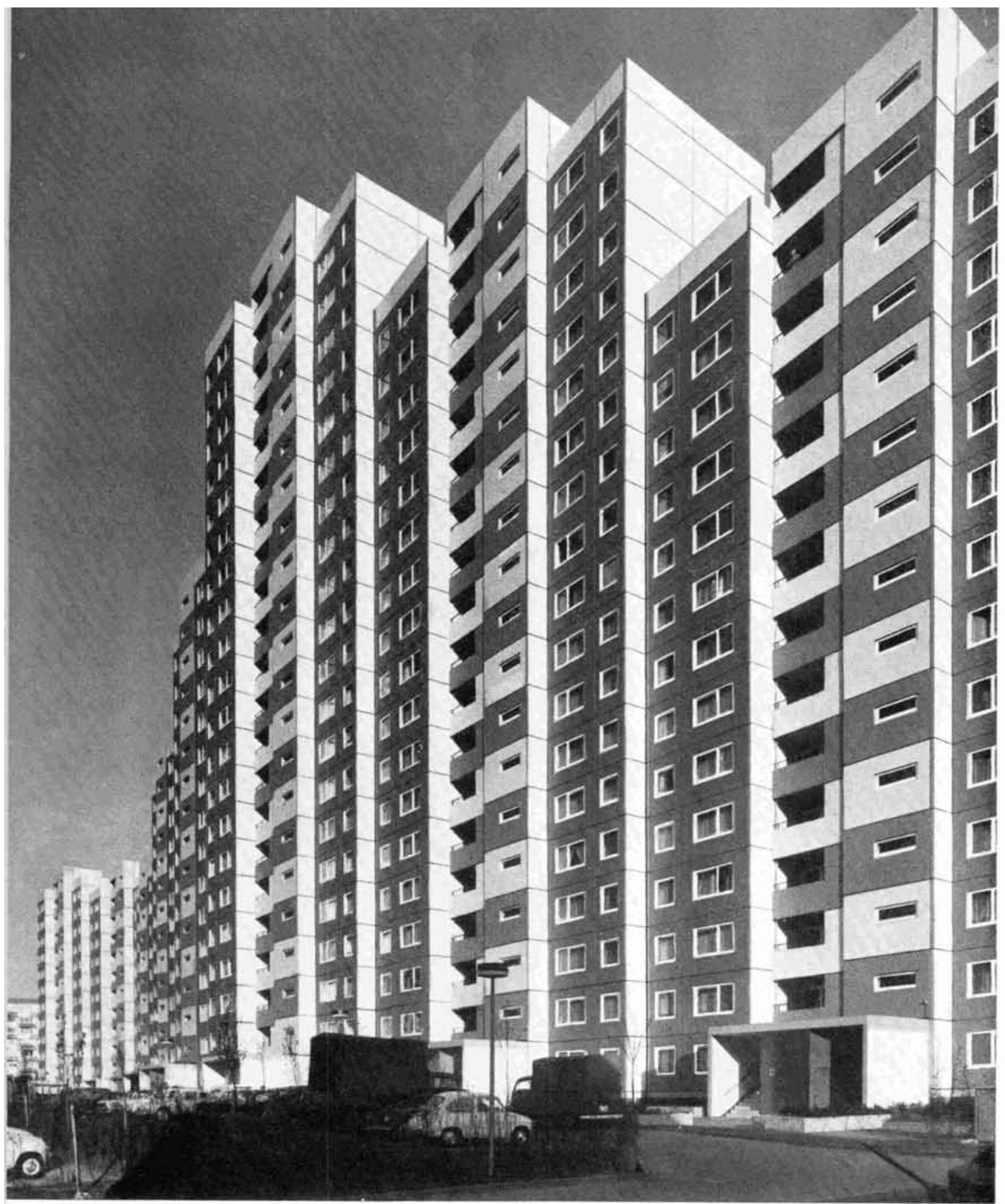

Hay que destacar la construcción de los balcones, concebidos y realizados como elementos colgados de las fachadas mediante ménsulas y elementos metálicos, así como el cuidado puesto, para que en las construcciones no se produjeran puentes térmicos, y que el entretenimiento de las instalaciones y su reparación pudiera efectuarse sin grandes molestias para los vecinos.

En la realización de este proyecto, el Profesor Trautwein ha demostrado, una vez más, que en todo tipo de construcciones, aun en las realizadas a base de elementos prefabricados, la dirección y coordinación del arquitecto es imprescindible.

Y que para la construcción de los futuros «habitats", las posibilidades que pueden ofrecer las construcciones prefabricadas, en cuanto a disminución de costos, variedad estética, etc., no han sido todavía agotadas. 


\section{Groupe d'éclifíces à Hamboumg - Allemagne}

Architecte: Prof. F. Trautwein, Dipl. ingénieur, et R. Behn, architecte BDA

Cet ensemble de vingt et un édifices, qui comportent au total 1.061 logements, se compose de deux unités, dont l'une de 716 logements, construits selon le système de préfabrication "Barets", et l'autre, de 345 logements, selon le système "Camus". Ces deux systèmes utilisent des panneaux pleins -béton armé- pour les murs intérieurs et les planchers, et des panneaux type "sandwich" pour les façades.

Digne de mention est la construction des balcons, conçus et réalisés comme des éléments suspendus aux façades à l'aide de consoles et d'éléments métalliques. Il faut souligner également la variété des plans et des élévations, ainsi que l'extraordinaire dynamisme et mouvement de l'ensemble.

\section{Skyscrupers in Hamburg, Germmmy}

Architect: Prof. F. Trautwein, Dipl. Eng., \& R. Behn, BDA architect

This project consists of 21 buildings and 1061 flats, and is divided into two units. One of them contains 716 flats, constructed by the «Barets" prefabrication method, and the other 345 flats, for which the "Camus" system has been adopted. Both techniques make use of solid panels of rein. forced concrete for partition walls and floorings, and sandwich type panels for the outer walls.

The construction of the balconies is noteworthy, which consist of elements hanging from the outer walls by means of overhangs and cables. Another outstanding feature is the variety of plan and elevation designs, which give a dynamic quality to the total aspect of the buildings.

\section{Hockbaus in Hamburg - Deutseblamed}

Architekt: Prof. Dipl. Ing. F. Trautwein und R. Behn, Architekt BDA

Der errichtete Komplex -21 Häuser mit insgesamt 1.061 Wohnungen- besteht aus zwei Einheiten: eine mit 716 Wohnungen, die nach dem «Barets»-Fertigbausystem errichtet wurden; die andere mi 345 Wohnungen nach dem "Camus»-System; beide Systeme basieren auf massiven Platten - aus Stahlbeton - in den Zwischenwänden und Decken, sowie auf Platten im "Sandwich»-Stil für die Aussenwände.

Erwähnenswert ist die Konstruktion der Balkons, die als freitragende Elemente entworfen und durchgeführt wurden, an den Fassaden aufgehängt mit Hilfe von Konsolen und Metallelementen. Ebenfalls erwähnenswert ist die Verschiedenartigkeit der Grund- und Aufrisse, sowie die ausserordentliche Dynamik und Bewegung des Komplexes. 